\title{
A Utilização do Método Radar de Penetração no Solo (GPR) para detecção de vergalhões.
}

Pablo Uale de Carvalho Silva, IG/UnB

Copyright 2019, SBGf - Sociedade Brasileira de Geofísica

This paper was prepared for presentation during the $16^{\text {th }}$ International Congress of the Brazilian Geophysical Society held in Rio de Janeiro, Brazil, 19-22 August 2019.

Contents of this paper were reviewed by the Technical Committee of the $16^{\text {th }}$ International Congress of the Brazilian Geophysical Society and do not necessarily represent any position of the SBGf, its officers or members. Electronic reproduction or storage of any part of this paper for commercial purposes without the written consent of the Brazilian Geophysical Society is prohibited.

\section{Abstract}

In this paper, it will be analyzed the results obtained in a geophysical survey on concrete at the upper groundfloor of the UnB Geoscience Institute. There were three acquistion lines made with an antena of $2,6 \mathrm{GHz}$ of the Geophysical Survey Systems Incorporated (GSSI). To locate the iron mesh, the GPR (Ground Penetrating Radar) method was used, which follows the principle of electromagnetic wave reflection to acquire information related to the subsurface.

The data processing was performed using the software REFLEXw, using the filter subtract-mean (dewow), the time zero correction, the energy decay gain and the bandpass frequency filter. In the analysis of the radargram sections, were identified the rebars in the floor's structure interior through the presence of hyperbolic features, caused by the difraction of the electromagnetic waves in the data.

\section{Introdução}

O método utilizado para aquisição de dados foi o método GPR (Ground Penetrating Radar) ou Radar de Penetração do Solo que é muito utilizado no contexto urbano por causa do seu caráter não destrutivo, não invasivo, do baixo custo de operação e da rapidez nos levantamentos de campo (Reynolds, 1997)

No âmbito das estruturas de concreto, o método do GPR possui várias aplicações, dentre elas: determinação da espessura do concreto, detecção/identificação de falhas de concretagem na estrutura, detecção de barras de aço, análise de pavimentação de concreto, entre outros como mencionado em Rocha et al., (2017).

Para estudos geotécnicos utiliza-se uma antena com frequência no intervalo de $100 \mathrm{MHz}$ a $2,6 \mathrm{GHz}$. No presente trabalho a antena utilizada na aquisição possuía frequência de 2,6 GHz o que indica uma maior resolução de dados porém um alcance pequeno.

O levantamento de cunho geotécnico apresentado nesse trabalho, teve como objetivo a verificação da composição da armação de concreto no chão, ou seja, definir a quantidade de vergalhões de ferro por metro. Em estudos com o método GPR para tubulações são verificados que a presença destas são feitas através da presença de ondas difratadas caracterizadas por uma hipérbole e com respectivo valor de velocidade como em Pinto (2010). Analogamente podemos usar essa teria para vergalhões.

A aquisição foi realizada no andar superior do Instituto de Geociências da UnB. O Instituto de Geociências se encontra na Ala central do ICC - Instituto Central de Ciências.

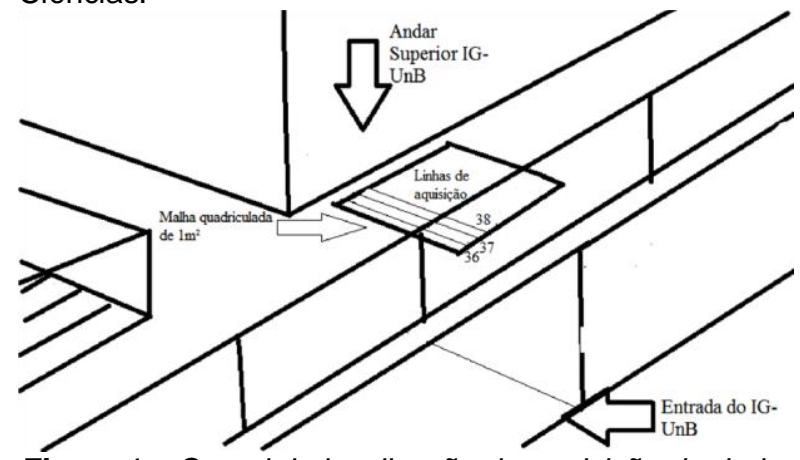

Figura 1 - Croqui da localização da aquisição de dados das três linhas do andar superior do IG-UnB

\section{Metodologia}

A aquisição de dados foi realizada no dia 30 de outubro do ano de 2014. O lugar da aquisição de dados foi o andar superior do IG-UnB, logo acima da entrada, do lado da escada. Os cabos do aparelho foram conectados e duas malhas quadriculadas de $1 \mathrm{~m}^{2}$ foram dispostas no chão. O equipamento utilizado foi um GPR da Geophysical Survey Systems Incorporated (GSSI) de 2,6 $\mathrm{GHz}$ (Figura 2).

Foram realizada três linhas na aquisição. Foram feitas em torno de 60 linhas. Os dados processados que estão presente nesse estudo de caso são referentes as linhas 37, 38 e 39. Nessa aquisição o número de amostras foram 256, e o intervalo temporal foi de 0,0390625 ns. Foram feitos 256 traços com espaçamento de 3 milímetros. È necessário atentar para o efeito do aliasing (falsa amostragem), logo esse parâmetro deve ser cuidadosamente escolhido. E janela temporal foi de 10 ns, sendo baixa devido a pequena profundidade que se tinha como alvo.

\section{Processamento dos dados}

No processamento foram utilizados filtros e ganhos para a identificação das hipérboles e como etapa final foi realizada uma migração com o objetivo de colapsar as difrações, fazendo com que a energia se concentre no topo da hipérbole. 


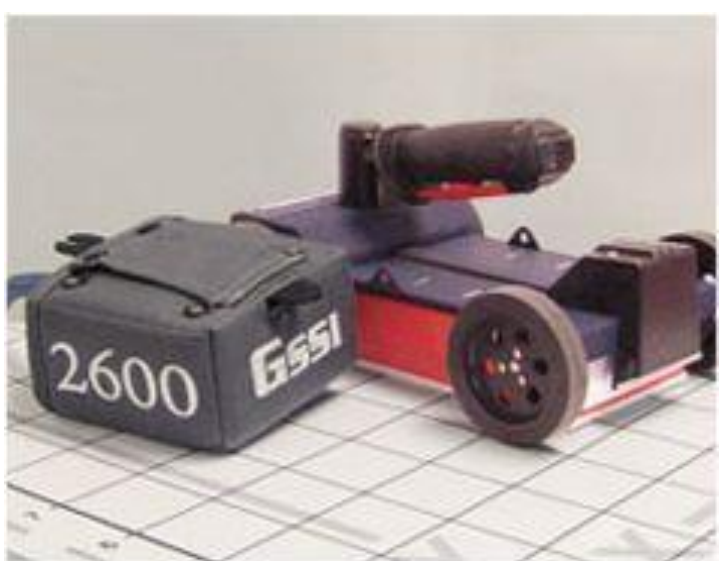

Figura 2 - Modelo do equipamento da GSSI 2,6 GHz utilizado para aquisição de dados em cima da malha quadriculada

Para processar os dados adquiridos, utilizou-se o programa Reflexw. Foi feita uma análise 2D pelo programa. $O$ formato de entrada para importar o arquivo foi o RADAN e o de saída o new 16 bit integer. A disposição das linhas é a several lines.

A rotina consistiu em utilização do filtro subtract-mean (dewow) com auxílio da janela WiggleWindow. Os respectivos tempos desse processamento foram:

Tabela 1 - Dados utilizados para o filtro 1D subtractmean (dewow)

\begin{tabular}{|l|l|l|}
\hline Linhas & Tempo inicial (ns) & Tempo Final (ns) \\
\hline 36 & 1,692409 & 2,79197 \\
\hline 37 & 1,60813 & 2,82810 \\
\hline 38 & 1,64234 & 2,73723 \\
\hline
\end{tabular}

Após a utilização desse filtro fez-se a correção do tempo zero, que também com auxílio da janela WiggleWindow e utilizou a opção de StaticCorrection, deixando no dado só as ondas refletidas por descontinuidades, e objetos da armação de concreto. Posteriormente foi utilizado um ganho do tipo energy decay e o filtro bandpassfrequency, os dados utilizados pro filtro estão dispostos na Tabela 2.

Há uma relação entre as distâncias desses pontos, que diz que a distância entre os dois primeiros pontos deve ser menor ou igual ao dobro da distância entre os dois últimos pontos.

\section{Discussão dos resultados}

Depois de ajustado os dados, as hipérboles foram marcadas, e os vergalhões localizados (Figura 4), (Figura 5) e (Figura 6). Apresentando como resultados diversos vergalhões dispostos na armação de concreto. As hipérboles foram identificadas com cautela, para que não fossem confundidas com "rabo de andorinha", termo utilizado para definir o encontro de duas hipérboles, o que pode confundir na hora do processamento.
Tabela 2 - Tabela que relaciona as linhas e os pontos utilizados para o filtro 1D band-passfrequency

\begin{tabular}{|l|l|l|l|l|}
\hline Linhas & $\begin{array}{l}\text { Low-cut } \\
\text { frequency } \\
(\mathrm{MHz})\end{array}$ & $\begin{array}{l}\text { Lower } \\
\text { Plateau } \\
(\mathrm{MHz})\end{array}$ & $\begin{array}{l}\text { Upper } \\
\text { Plateau } \\
(\mathrm{MHz})\end{array}$ & $\begin{array}{l}\text { High-cut } \\
\text { frequency } \\
(\mathrm{MHz})\end{array}$ \\
\hline 37 & 289,1841 & 795,2562 & 1397,723 & 3590,702 \\
\hline 38 & 433,7761 & 795,2562 & 2000,19 & 3446,11 \\
\hline 39 & 530,1708 & 891,6589 & 1566,414 & 3012,334 \\
\hline
\end{tabular}

Nas seções de radar foram possível observar algumas feições hiperbólicas, indicadas pelos ápices luminoso devido as etapas do processamento. Eles se encontram na altura $0,1 \mathrm{~m}$ nas linhas 37 e 38 e um pouco mais raso na linha 39 , em torno de $0,08 \mathrm{~m}$ do início do perfil. Nesses perfis também é possível um forte refletor horizontal contínuo situado a aproximadamente $0,23 \mathrm{~m}$ indicando o fim do concreto, no caso o contato concreto-ar.

Nota-se que na linha 37 (Figura 6) os vergalhões não estão equiespaçados, há espaçamentos de $0,03 \mathrm{~m}, 0,04 \mathrm{~m}$ e $0,05 \mathrm{~m}$. Na linha 39 (Figura 5) temos três vergalhões equiespaçados em $0.08 \mathrm{~m}$ e o primeiro vergalhão a $0,22 \mathrm{~m}$ do segundo. Na linha 38 (Figura 4) é possível verificar um padrão espaçamento de aproximadamente $0,05 \mathrm{~m}$ entre os três últimos vergalhões e os dois primeiros. Logo é perceptível que não há um padrão na armação de ferro no chão. As diferenças são de poucos centímetros, sendo considerado comum, devido a não minuciosidade na hora de armar a malha de ferro para que os vergalhões fiquem igualmente espaçados.

A velocidade da onda na linha 37 foi de $0,1385 \mathrm{~m} / \mathrm{ns}$, na linha 38 foi de $0,14625 \mathrm{~m} / \mathrm{ns}$ e na linha 39 foi de $0,093225 \mathrm{~m} / \mathrm{ns}$. Os valores de velocidade da linha 37 e 38 foram os que mais se aproximaram do valor da velocidade da onda eletromagnética no concreto seco, que é $0,127792 \mathrm{~m} / \mathrm{ns}$ segundo cálculos utilizando os dados de Porsani (1999) O valor da linha 39 se assemelha mais a velocidade da onda eletromagnética no concreto saturado. A linha 39 apresentou problemas devidos a imprevistos na aquisição associados a travação do carrinho do GPR, gerando um impulso que resultou em uma coluna de descontinuidade no dado, resultando num dado com menos hipérboles e com uma velocidade menor que as outras.

\section{Conclusões}

A prática resultou em um maior entendimento da teoria do método, fez com que houvesse a interligação entre teoria e o que é realmente cobrado em uma situação de aquisição de dados geofísicos do método GPR.

Pode-se concluir que na estrutura do chão do andar superior do IG-UnB possui vergalhões dispostos um ao lado do outro de modo que consegue-se ver até pelo menos 7 hipérboles, indicando 7 vergalhões dispostos no concreto. Comparando os dados de todos as linhas há um padrão de pelo menos 7 hipérboles por metro no piso do andar superior do IG. 
Confirma-se assim que para levantamentos do método GPR de cunho geotécnico, antenas de altas frequências são viáveis e obtém-se resultados bastante positivos, afirmando a veracidade e confiabilidade do método de GPR.

\section{Agradecimentos}

Agradecimentos ao professor Welitom Borges Rodrigues que emprestou o equipamento para realização do trabalho, e Juliana Teles Diniz Gonçalves que revisou o trabalho.

\section{Referências}

REYNOLDS, J. M. (1997). An Introdution to Applied and Environmental Geophysics. John Wiley \& Sons. 796 p.

PINTO, G. P. 2010. O Método GPR aplicado a localização de tubulações utilizadas no (Mestrado em Geofísica) - Programa de Pós-Graduação em Geofísica, Universidade Federal do Pará, Belém, 2010.abastecimento de água na região urbana do município de Belém-Pará. Dissertação.

ROCHA, P. H. et al. 2017. Utilização do Radar de Penetração no Subsolo (GPR) na caracterização de armaduras em estruturas de concreto armado. Apresentado no IV Congresso de Ensino Pesquisa e Extensão da UEG.

PORSANI, J.L.,1999a. Ground penetrating radar (GPR): Proposta metodológica de emprego em estudos geológico-geotécnicos nas regiões de Rio Claro e Descalvado - SP. Tese de Doutorado, Instituto de Geociências e Ciências Exatas, UNESP, Campus de Rio Claro - SP. 145p. 


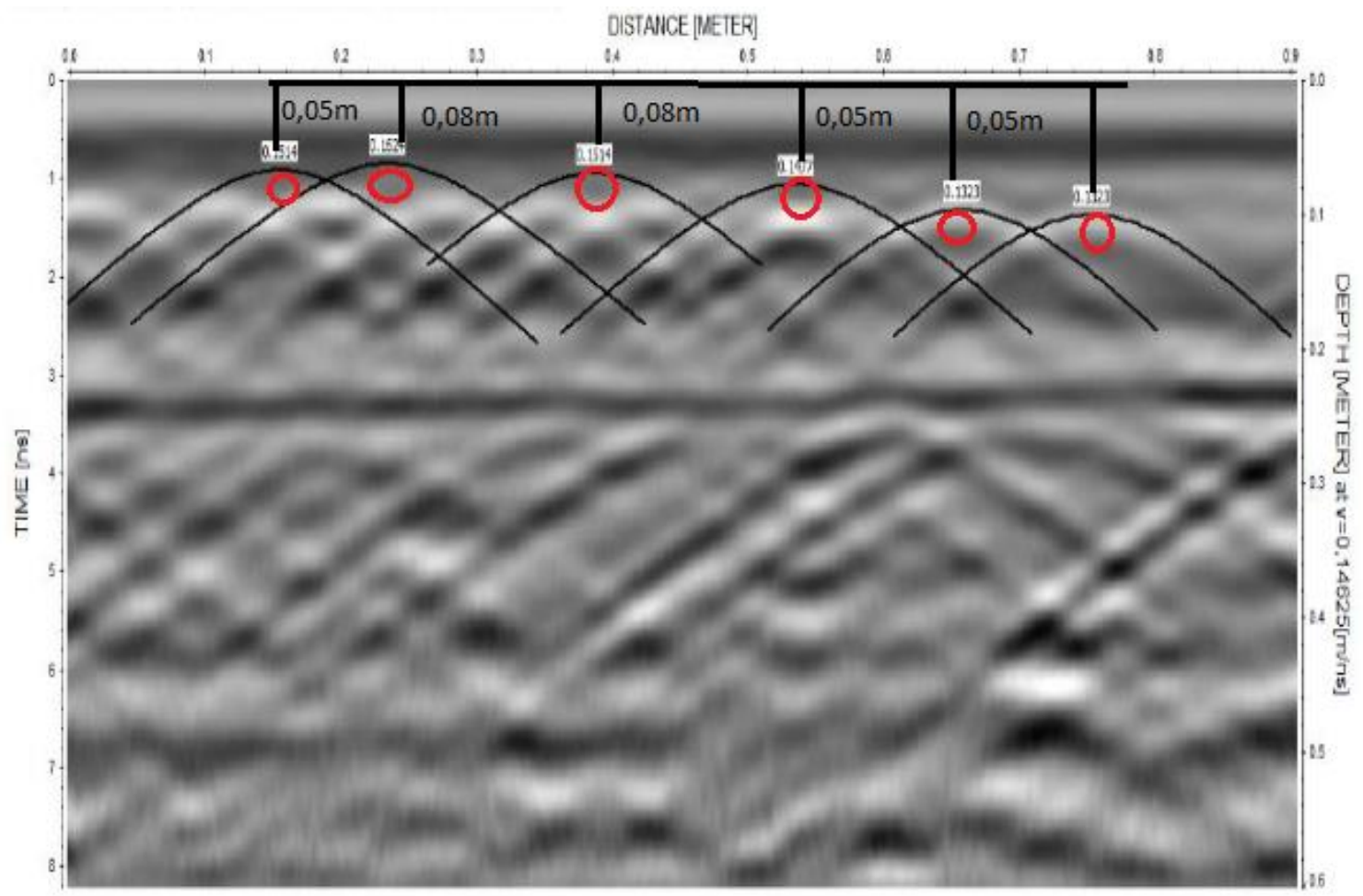

Figura 4 - Linha 38

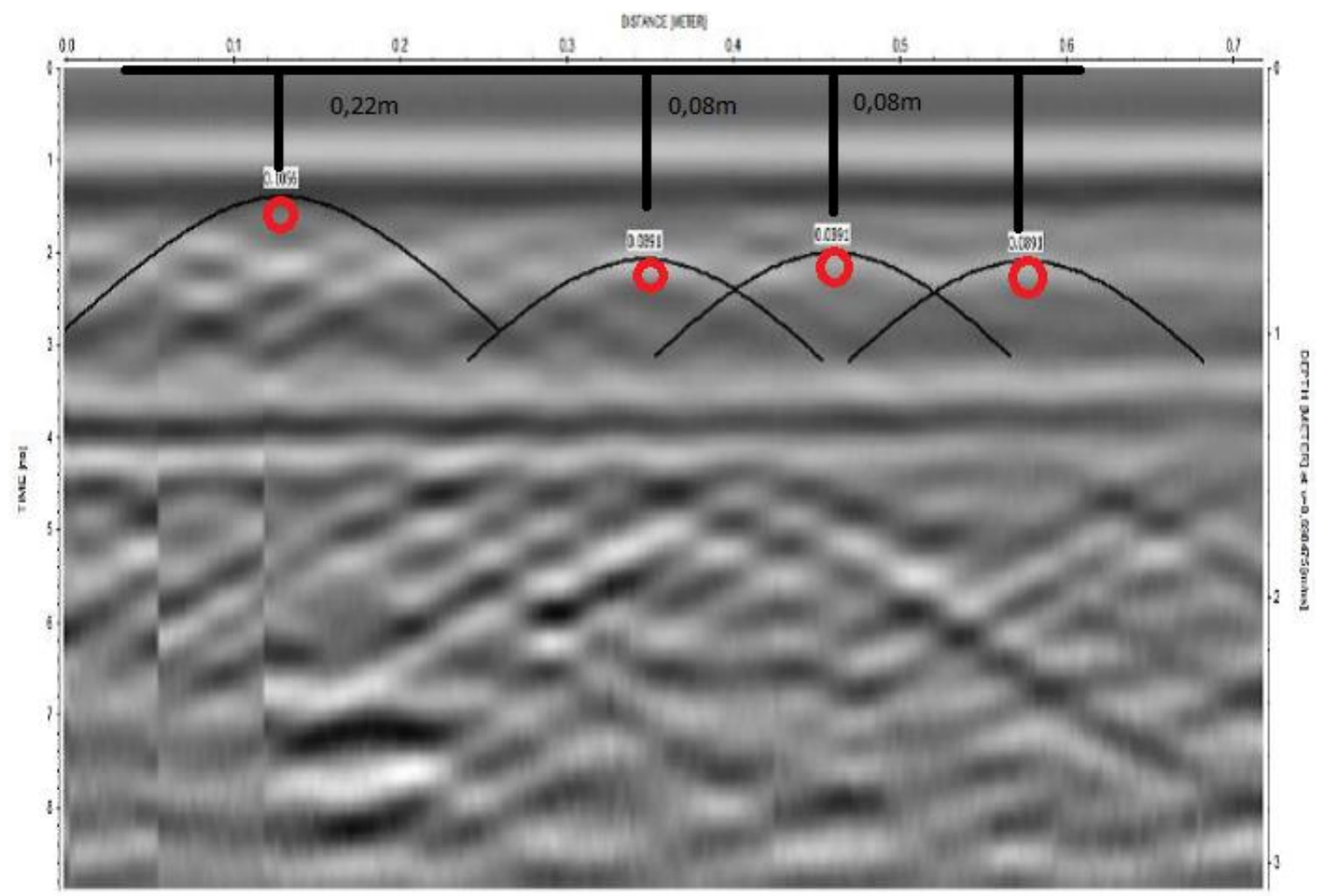

Figura 5 - Linha 39 


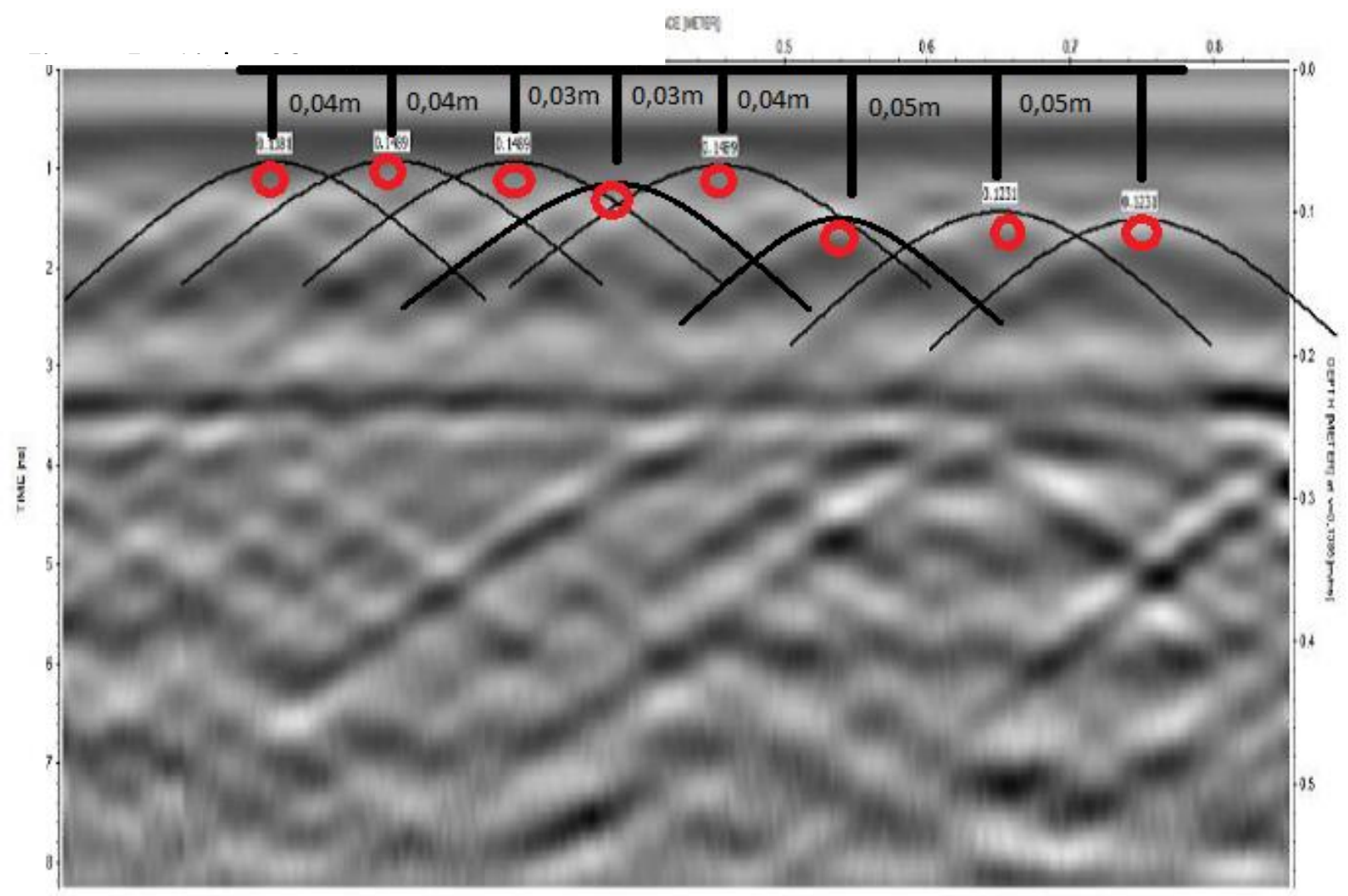

Figura 6 - Linha 37 